\title{
Fortbildungskontrolle in Seldwyla
}

Felix Tapernoux

1 Hänggeli C, Bauer W, Gähler E. Update Fortbildung: Wozu benötige ich ein Fortbildungsdiplom? Schweiz Ärztezeitung. 2012;93(26):987-90.

2 Binswanger RO. Outcomestatt Prozessorientierung in der ärztlichen Weiterbil dung. Schweiz Ärztezeitung. 2012;93(4):132.

Korrespondenz:

Dr. med. Felix Tapernoux Ferrachstrasse 29

CH-8630 Rüti

ftapernoux[at]aol.com
Kürzlich haben uns die Kollegen des SIWF wieder einmal an unsere Fortbildungspflicht erinnert [1]. Bezeichnenderweise heisst der Titel diesmal «Wozu benötige ich ein Fortbildungsdiplom?» und nicht «Wozu brauche ich Fortbildung?» Was nützen also Fortbildungen ohne Diplome? Zwar schreiben die Kollegen in ihrem Fazit: «Lebenslange Fortbildung ist für eigenverantwortlich tätige Ärztinnen und Ärzte eine Selbstverständlichkeit», doch man scheint dieser Eigenverantwortung doch nicht recht $\mathrm{zu}$ trauen, sonst müsste man nicht vorher ein immer enger werdendes «freiwilliges» Zwangskorsett vorstellen in einem Konstrukt, das nicht nur eine Eigendynamik entwickelt hat, sondern zu einem vermutlich lukrativen Institut angewachsen ist.

Wenn man die jahrelangen Bemühungen in diesem Bereich von den Anfängen des Kinderarztes Dr. R. Salzberg verfolgt hat, ging es stets darum, einer staatlichen Regulierung zuvorzukommen und gegen aussen zu beweisen, dass man sich "fortbildet», und dies auf eine Weise, die nichts mit Qualität zu tun hat. (Schein ist eben wichtiger als Sein, vgl. Erich Fromm, «Haben oder sein», 1976). Dass man heute in der Industrie stets von prozessorientierter Qualität spricht, was inzwischen auch im ganzen Bildungssektor Einzug gehalten hat und sich eigentlich niemand mehr für den Outcome interessiert, ist auch unserer «Fortbildung» zugute gekommen. Einmal in die falsche Richtung angestossen, wird sie zunehmend perfektioniert. Das Sammeln von Credits bzw. das Absitzen von Fortbildungen wie im Konfirmandenunterricht wird mit Qualität gleichgesetzt. «Zwangsabsitzen», um den «heutigen Stand des Irrtums» zu erfahren, lässt an die ehemalige DDR denken, wo man über die Fortschritte des Kommunismus öffentlich (des)informiert wurde. (Ob nun dieser Marx bewohnt sei, wollte am Schluss ein «aufmerksamer» Zuhörer wissen!). An einer Fortbildung über Verkehrsmedizin, die von vielen emeritierten Kollegen besucht wurde, konnte ich erleben, wie diese sich am Schluss nach vorne drängten, um ihr Diplom zu erhalten. Ob sie ihre Credits und Diplome wohl an der Himmelstüre vorweisen wollen?

Nun erfolgt noch die wirklich niederschmetternde Quintessenz für alle KollegInnen, die ihr Wartezimmer mit Diplomen verunstaltet haben: Man kann das Fortbildungsdiplom selber ausdrucken. Welche Demütigung für einen echten Diplomsammler! Man stelle sich nur eine Praxis vor, tapeziert mit selbstgedruckten Diplomen.

Bemerkenswert, dass Kollege Werner Bauer, der in seinen stets interessanten Beiträgen jeweils William Osler (1849-1919) zitiert, unter die Erbsenzähler ge- gangen ist. Was würde wohl Osler über diese Art heutiger Fortbildungskontrolle in der Schweiz sagen? Kollege Bauer meint mit dem Zitat «Iflicense to practise meant the completion of his education how sad it would be for the practitioner, how distressing for his patients! More clearly than any other the physician should illustrate the truth of Plato's saying that education is a life-long process» (W. Osler, Lancet, 1900), Osler als frühen Anhänger der «Kontrollitis» bzw. «Verschulung der Gesellschaft» vereinnahmen zu dürfen. Mit Toten und deren Zitaten darf man ja bekanntlich alles machen, nur redlich und wissenschaftlich ist das nicht.

Kürzlich hat Kollege Binswanger [2] die heutige prozessorientierte Fortbildung kritisiert und sich für einen Wechsel zur Outcome-Orientierung ausgesprochen. Eigentlich waren wir älteren Semester stets auf Outcome gezüchtet worden und Prüfungen, so partikular sie Wissen/Fähigkeiten beweisen, haben ein ganz anderes Niveau als «Dabeisein und Sammeln». Wie fortschrittlich dies entgegen dem heutigen Zeitgeist sein könnte. Ich habe schon zu Beginn dieser lächerlichen Credit-Sammlerei stets gedacht, wie viel sinnvoller wäre es, wenn jeder Arzt einmal pro Jahr nur eine in diesem Jahr erfahrene Neuerung mitteilen würde, die er in seinen Praxisalltag integriert hat. Ein Nachhaltigkeitsbeweis! Die besten Antworten könnte

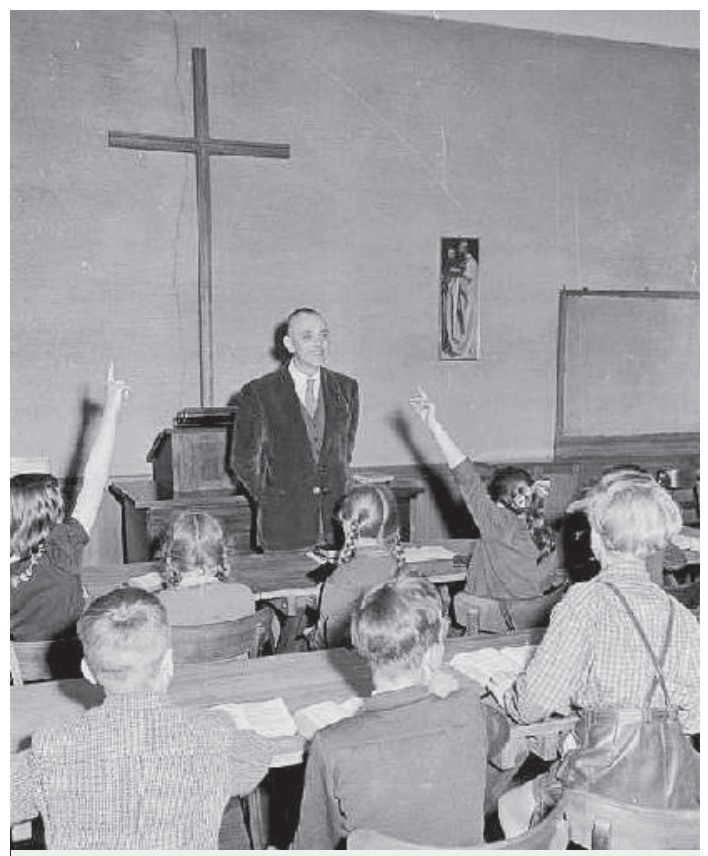

Die Art der ärztlichen Weiter- und Fortbildung erinnert den Autor an seinen Konfirmationsunterricht. (Bundesarchiv, Bild 194-1358-29A / Lachmann, Hans / CC-BY-SA) 
man dann publizieren, und dies würde jedenfalls mehr zur Qualität beitragen. Selbst eine Prüfung jedes Jahr über ein vorgegebenes Thema wäre eine intellektuelle Leistung, die weit über dem aktuellen Niveau stünde. Ausserdem ist es evident, dass sich die meisten Ärzte auf ihrem eigenen Gebiet à jour halten, auch ohne Zwang und (Selbst-)Kontrolle. Von einigen allzu spezialisierten Kollegen müsste man eher fordern, sich jeweils in Nachbargebieten ein bisschen kundig zu machen.

Geht es darum, die paar Fortbildungsunwilligen zu erfassen und an den Pranger zu stellen? Nein, es geht darum, auch erwachsene Menschen ein Leben lang zu disziplinieren, zu regulieren, mit unsinnigen Richtlinien zu beschäftigen, an irgendwelche Normen anzupassen, konform und gleich zu machen. Wie viel sinnlose Zeit und welche Kreativität dadurch verlorengeht! Die Aufklärung hat sich leider nie durchgesetzt, paradoxerweise am wenigsten im Bildungswesen: «Der Ausgang des Menschen aus seiner selbstverschuldeten Unmündigkeit» (Immanuel Kant, 1724-1804).

Inwischen habe ich fast alle meine Dignitäten (Besitzstandleistungen), die ich ohnehin nie verwendet habe, wegfallen lassen. Geblieben sind einige wenige wie Entfernen eines subkutanen Prozesses bzw. Fremdkörpers. Nach einem Jahr Chirurgie und einem Jahr Dermatologie und 20 Jahren Praxis traue ich mir das zu ohne Absitzen einer entsprechenden Fortbildung. Im Notfalldienst musste und muss ich stets Kinder unter 6 Jahren untersuchen, oftmals nachts. Den Mentalstatus prüfe ich regelmässig bei der Fahrtauglichkeitsabklärung, mache sogar den Trail-Making-Test A und B, habe auch Fortbildungen über Demenz besucht, vielleicht nicht in den letzten zwei vergangenen Jahren. Nun soll ich dies nicht mehr zuhanden der Krankenkasse abrechnen dürfen ohne lächerliches Prozedere. Dies ist das Resultat einer verfehlten Fortbildungspraxis!

Zahlreiche Kollegen haben bereits Kritik an der heutigen Fortbildungspraxis geübt [3, 4]. Sie alle sind Rufer in der Wüste geblieben. Ebenso wie die grundsätzlicheren Kritiker der Verschulung der Gesellschaft (es sei hier an Everett Reimer und Ivan Illich erinnert), die unaufhörlich weiterschreitet und eigentlich nur eines hervorbringt: absolute Mittelmässigkeit. Inzwischen ist die ganze Welt zu einem Dorf wie Seldwyla geworden. Typischerweise haben die meisten Genies sich der vorgegebenen Schulung oder Fortbildung verweigert. Als jüngstes eindrückliches Beispiel sei hier an Steve Jobs und seine Biographie bzw. die eindrückliche Rede vor Maturanden erinnert. Aber wir lernen nichts von solchen Genies. Anstatt erwachsene Akademiker endlich in die Eigenverantwortung $\mathrm{zu}$ entlassen, muss man ein Leben lang irgendwelchen selbsternannten Experten beweisen, dass man sein «Handwerk» beherrscht. «Vertrauen ist gut, Kontrolle ist besser», auch wenn es bisher noch eine Art «Selbstkontrolle» über abgesessene und halbschla- fend konsumierte Vorträge darstellt. Aber abwarten: Die Schrauben werden schon noch angezogen! Und das vermutlich von Politikern, die sich nie einer Qualitätsprüfung unterzogen haben und wie kürzlich stets beweisen, dass sie völlig an der Bevölkerung vorbeipolitisieren, speziell im Gesundheitswesen. Es muss nur etwas Druck von dieser Seite kommen, und schon sind die «Erbsenzähler» bereit, diesem nachzugeben.

Über Qualität und Qualitätsmessung in der Medizin müsste man einen eigenen Bericht verfassen. Aber Fortbildung und Qualität sind in den Augen der «Verschulungsanhänger» wie siamesische Zwillinge, da man menschliche Qualitäten und Sozialkompetenz nicht messen kann, aber die Anzahl besuchter Fortbildungen und die Diplome an den Wänden. Und man passt sich eben dem Zeitgeist an. Man wird jedoch den Eindruck nicht los, dass sich die wahre Qualität umgekehrt zur Anzahl an aufgehängten Diplomen, grossspurigen Internetauftritten und selbsternannten Titeln verhält. Gegenseitige Sympathie und Vertrauen stellen sich meist bei der ersten Begegnung ein und sind mindestens so wichtig wie scheinbar messbare Qualität. Es ist nicht zufällig, dass ManagedCare-Netzwerke die Qualitätsdiskussion massgeblich $\mathrm{zu}$ ihren Zwecken beeinflusst haben und z.B. einen Titel «Mehrfacharzt» geschaffen haben [5]. Gewissen Kollegen genügt auch der Professorentitel nicht mehr, weshalb sie ein «Center of Excellence» bilden. Bei uns an der Basis häufen sich Klagen der Patienten, während die Spitäler mittels Umfragen sich stets mit einer überwiegenden Zufriedenheit schmücken können. Die Unzufriedenen bilden dann immer die Ausnahme, für die es keine Anlaufstelle ausser uns und die Patientenorganisationen gibt. Spitäler sind bis heute nicht bereit, eine Ombudsstelle für Reklamationen einzurichten. Und solche Reklamationen an die jeweiligen Chefärzte weiterzuleiten, wird uns «Kleinen» rasch verleidet, da man weder im Leben noch an einer Fortbildung gelernt hat, rein problembezogen mit Fehlleistungen umzugehen.

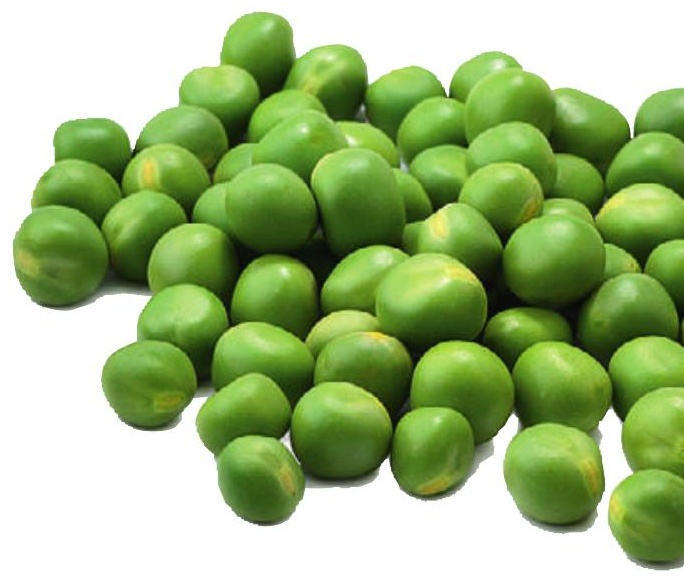

Nun, liebe Kollegen Erbsenzähler: Macht endlich Nägel mit Köpfen, prüft das Resultat von Fortbildungen oder lasst es ganz bleiben! 